\title{
Thoracoscopic Repair of Congenital Diaphragmatic Hernia, First Ever Study from Pakistan
}

\author{
Ali Raza Brohi ${ }^{1 *}$ and Naseem Mengal $^{2}$ \\ ${ }^{1}$ Professor and Chairman, Paediatric Surgery, Peoples University of Medical and Health Sciences, Nawabshah, Pakistan \\ ${ }^{2}$ Assistant professor, Paediatric Surgery, Peoples University of Medical and Health Sciences, Nawabshah, Pakistan \\ *Corresponding Author: Ali Raza Brohi, Paediatric Surgery, Peoples University of Medical and Health Sciences, Pakistan.
}

Received: July 23, 2019; Published: August 13, 2019

\begin{abstract}
Key words Hernia, Diaphragmatic, Thoracoscopic, congenital

Purpose of study: To analyze indications and to present demographic data, details of surgical technique and outcomes of thoracoscopic repair for congenital diaphragmatic hernia.

Methodology: This is retrospectively study conducted at one of the medical university of Pakistan and we reviewed medical records of 37 patients with CDH who underwent thoracoscopic repair by the same surgical team from March 2013 to June 2017. The operations were performed using three trocars one $5 \mathrm{~mm}$ for camera below scapula at 5 th ICS and two $3 \mathrm{~mm}$ working ports one space below camera port, one at posterior axillary line and other $3 \mathrm{~mm}$ working port between tip of scapula and vertebral line. Pleural insufflation with carbon dioxide was maintained at a pressure of 4-6 mm Hg. The hernia defect was repaired using nonabsorbable-interrupted intracorporeal suturing with $2 / 0$ silk or $2 / 0$ ethibond. A prosthetic proline mesh was used in 4 patients when primary closure of the hernia defect was not feasible.

Results: There were 37 patients, including 13 boys and 24 girls. 12 patients were newborns, 17 patients were infants 8 above infantile age. The hernia was located on the left side in 31 (83.78\%) patients and on right side in 6 patients (16.21\%). The operative time for thoracoscopic approach was between 80 to 150 minutes. 15 patients required mechanical ventilator support. Conversion was done in one patient who was not maintaining saturation. Follow-up was obtained in $29(78.37 \%)$ patients ranging from 1 to 6 months. 1 patient had recurrence that required open redo repair.

Conclusions: Thoracoscopic repair is feasible and safe for neonates and infants with CDH. Our initial results are better because majority of patients were not neonates and others presented a little later in infancy and in stable condition.
\end{abstract}

Keywords: Thoracoscopic; Diaphragmatic Hernia; Pakistan

\section{Introduction}

Congenital diaphragmatic hernia (CDH) occurs in every 1 in 2500-4000 live births [1]. In 1995, Silen., et al. performed first thoracoscopic repair in adolescent [2] and in same year laparoscopic repair of CDH in 6-month-old female was also reported [3]. Becmeur., et al. in 2001 reported their first experience of thoracoscopic CDH repair on 8.3-month-old infant [4]. In Pakistan many centers have started minimal invasive surgery but still they are in their initial phases and this is the first study, which is reported from Pakistan. In this article, we are reporting our experience of 37 cases and the purpose of study is to share our initial surgical experience, outcomes and complications of thoracoscopic $\mathrm{CDH}$ repair.

\section{Materials and Methods}

After approval from ethical committee of university we reviewed records of patients who had been operated thoracoscopi- cally for CDH by single surgeon from March 2013 to June 2017 at one of the medical university of Pakistan. In all patients, $\mathrm{x}$-ray chest, echocardiography, CT chest and ABGs were done. Those neonates who were having severe cardiac abnormality, weight less than 2 $\mathrm{kg}$, severe pulmonary hypertension and pre-operatively unstable even on mechanical ventilator support were not selected for thoracoscopic repair. Patients followed for 6 months, initial follow-up was at first week after discharge, then every month. In follow-ups clinical examination and $\mathrm{x}$-ray chests were done along with general status and growth of patient.

\section{Surgical technique}

General Anesthesia with bilateral ventilation is used in all cases. Patient placed in lateral decubitus position with upper arm elevation. Surgeon stands at patient head, monitor positioned at patient feet. First trocar of $5 \mathrm{~mm}$ for thoracoscope is introduced below tip 
of scapula at 5th intercostal space (ICS). Carbon dioxide insufflation started at $3 \mathrm{mmHg}$ pressure, which is increased up to max $6 \mathrm{~mm} / \mathrm{hg}$ if there is difficulty in content reduction. Second trocar of $3 \mathrm{~mm}$ placed in between vertebral line and tip of scapula at $6 \mathrm{th}$ intercostal and third trocar of $3 \mathrm{~mm}$ placed in posterior axillary line at 6 th intercostal space but on right side hernia the trocar placement was one space high because of presence of liver in chest (Figure: 1 port positions).

After visualization and orientation of contents gentle reduction was done easily except liver and spleen were difficult to reduce. The hernia defect repaired by using interrupted non- absorbable sutures (2/0 Silk or Ethibond) always taking first stitch from middle of defect and all knots tied intracorporeally. In those cases, where defect was large proline mesh used that is tacked with diaphragm rims in interrupted stiches of $3 / 0$ Ethibond and where the defect was wide at posterolateral end peri costal stitch taken and tied outside underneath the skin. Those hernias with sac the defect was repaired along with sac. Chest tube kept in all cases. (Figure: 2 procedure steps and 3 peri costal stich)

\section{Results}

There were 37 patients who had been operated thoracoscopically in which 12 were neonate, 17 infants and 8 patients were above infantile age (one patient operated at age of 8 years). Thirteen patients were boys and 24 girls. Average operative time for thoracoscopic repair was ranging between 80 to $150 \mathrm{mins}$.

In $31(83.7 \%)$ cases, defect was on left side and 6 (16.2\%) patients had right side defect. Hernia sac was present in 8 patients and absent in 29 patients. In all 31 patients with left side defect, apart from small and large gut there was spleen in 14 cases and stomach in 6 cases. While on the right side liver was main content in all 6 patients along with sac in 3 patients.

The defect repaired primarily in 33 patients and proline mesh was used in 4 patients with large defect. In 4 patients where there was deficient diaphragm rim at most lateral part of defect so peri costal stitch taken and tied outside under the skin to close the defect. During operation we observed hernia sac in 8 patients (5 left and 3 right side), excision of sac wasn't done instead it is plicated along with defect repair.

Conversion done in one case that was not maintaining saturation during surgery. Postoperatively, 15 patients required mechanical ventilation supports out of which two patients expired during ventilator support because of pulmonary hypertension. Besides this the other complication observed were pleural effusion
(1), atelectasis (2), and port site infection (1) sub-acute intestinal obstruction (1). Peri costal stitch infection was observed in 2 patients where silk suture used. In one patient recurrence occurred because of mesh infection, which required redo open surgery. Most of the problems, dealt conservatively except recurrence. Follow-up was obtained in 29 patients (78.37\%) ranging from 1- 6 months, 8 patient lost from follow-up. (Figure: 4 X-rays and peri costal stich abscess)

\section{Discussion}

After 1995, many pediatric surgeons shared their experience with minimal invasive approaches and have adopted different techniques of patient positions, trocar placements and their technique of doing procedures. Thoracoscopic approach is more feasible then laparoscopic approach, as there is a natural working space because of hypoplastic lung. Ferreira and Kim., et al. have technique of placing their first trocar below the tip of scapula, other working ports in 4 th or 5 th intercostal space. Liem., et al. placed trocars in higher positions 2nd or 3rd intercostal space for thoracoscope, other two trocars in 4th intercostal space [5-7].

But in our study, because of variable age of children operated, we have placed primary ports in 5 th intercostal space $(5 \mathrm{~mm}$ trocar) and secondary ports in 6th intercostal space (3mm trocars). Reason is to perform intracorporeal suturing easily in every age group, which will be difficult if we place trocars in higher space.

The content reduction was difficult in neonates, because of small cavity. Initial reduction was always difficult when chest is full of intestine. Thoracoscopic view for reduction of content is easier, but there is always chance of damage and bleeding so gentle reduction is always required and where the spleen was content mostly it is reduced at the end as it provides shield over the defect and helps in repair [5].

Apart from better visualization of defect by thoracoscopy there are other various advantages like superior cosmetic, less narcotic used postoperatively and long term musculoskeletal and spinal deformities [8].

In our study, out of 33 cases of primary repair we had repaired the defect with silk $2 / 0$ in initial 13 cases while in rest of the 20 we used Ethibond 2/0 compare to other studies surgeons who have used different suture material like proline for the repair of defect [9].

The right side defects are always wide and technically difficult to repair, as compared to left side, but thoracoscopic approach is 
more feasible than laparoscopy as it is difficult to bring liver down and difficult to place sutures as defect can't be visualized properly from below. Where the sac was present it is easy to repair the defect and same was observed by Liem., et al. [10].What we have modified in those patients who were having hernia sac, it wasn't excised instead of that plication was done which brought the defect closer and over that we repaired the actual muscular defect.

Different surgeons used different types of mesh in their studies $[11,12]$ but we used proline mesh in 4 cases $(10.8 \%)$ with large defect especially those on right side, as it is cheap and readily available in our institute.

Longer operative time is a common issue in minimal invasive procedures but with time when surgeon gets expertise it becomes shorter. The average operative time in our series ranges between 80 -150 minutes that is comparable with other reports in literature $[13,14]$.

It had been reported earlier in literature about high conversion rates for $\mathrm{CDH}$ repair by minimal Invasive approach that ranges from $3.4 \%$ to $14 \%[15,16]$ but in our study, we did conversion in only 1 patient (2.7\%), as child was not maintaining saturation and the other reason for less recurrence might be our strict selection criteria for thoracoscopic $\mathrm{CDH}$ repair.

In our study there were no intraoperative death, only 2 neonates expired that were on mechanical ventilator died because of pulmonary hypertension. Besides this overall complication rate is $21.6 \%$ that is quite low as compare to Cho., et al. reported in his study i.e.55\% [15]. One important complication which we observed in late follow-ups was peri costal stitch infection in $2.7 \%$ patient because of silk suture so we avoided using silk for peri costal suturing instead now we are using ethibond and this problem has not been reported in literature.

It had been debated in earlier studies that recurrences are higher (ranging from 5-40\%) following thoracoscopic repair than open repair [5,15,17,18]. In 2017 Tyson., et al. [19] in his study reported zero recurrence rate in thoracoscopic cohort and Liem., et al. reported $3.6 \%$ recurrence rate [7]. In our study we observed only $2.7 \%$ recurrence rate. In 2015, a survey done on IPEG members they reported that $89 \%$ surgeons are performing thoracoscopic $\mathrm{CDH}$ repair and $50 \%$ surgeons were in opinion that $\mathrm{CDH}$ repair can be done thoracoscopically despite of $57 \%$ recurrence rate [20].

\section{Conclusion}

Thoracoscopic repair is feasible and safe for neonates and infants with $\mathrm{CDH}$. Our initial results are better because majority of patients were not neonates and presented a little later in infancy and in stable condition. Results in our study may be better as our selection criteria was safe and the recurrence was less as our follow-up period was short.

\section{Bibliography}

1. Langham JM., et al. "Congenital diaphragmatic hernia. Epidemiology and outcome". Clinics in perinatology 23.4 (1996): 671-688.

2. Silen ML., et al. "Video-assisted thoracic surgical repair of a foramen of Bochdalek hernia". The Annals of thoracic surgery 60.2 (1995): 448-450.

3. Van der Zee DC and Bax NM. "Laparoscopic repair of congenital diaphragmatic hernia in a 6-month-old child". Surgical Endoscopy 9.9 (1995): 1001-1003.

4. Becmeur F., et al. "Thoracoscopic treatment for delayed presentation of congenital diaphragmatic hernia in the infant". Surgical Endoscopy 15.10 (2001): 1163-1166.

5. Ferreira CG., et al. "Neonatal minimally invasive surgery for congenital diaphragmatic hernias: a multicenter study using thoracoscopy or laparoscopy". Surgical endoscopy 23.7 (2009): 1650-1659.

6. Kim AC., et al. "Thoracoscopic repair of congenital diaphragmatic hernia in neonates: lessons learned". Journal of Laparoendoscpic and Advanced Surgical Techniques 19.4 (2009): 575-580.

7. Liem NT., et al. "Thoracoscopic repair for congenital diaphragmatic hernia: experience with 139 cases". Journal of Laparoendoscpic and Advanced Surgical Techniques 21.3 (2011): 267270.

8. Lawal TA., et al. "Thoracoscopy versus thoracotomy improves midterm musculoskeletal status and cosmesis in infants and children". The Annals of thoracic surgery 87.1 (2009): 224-228.

9. He QM., et al. "Standardized indications to assist in the safe thoracoscopic repair of congenital diaphragmatic hernia in neonates". Journal of Laparoendoscpic and Advanced Surgical Techniques 26.5 (2016): 399-403.

10. Liem NT., et al. "Thoracoscopic repair for right congenital diaphragmatic hernia". Journal of Laparoendoscpic and Advanced Surgical Techniques 18.4 (2008): 661-366.

11. Guner YS., et al. "Thoracoscopic repair of neonatal diaphragmatic hernia". Journal of Laparoendoscpic and Advanced Surgical Techniques 18.6 (2008): 875-880. 
12. Huang JS., et al. "Thoracoscopic repair of congenital diaphragmatic hernia: two centers' experience with 60 patients". Pediatric surgery international 31.2 (2015): 191-195.

13. Nam SH., et al. "Shifting from laparotomy to thoracoscopic repair of congenital diaphragmatic hernia in neonates: early experience". World journal of surgery 37.11 (2013): 2711-2716.

14. Tanaka T., et al. "Surgical intervention for congenital diaphragmatic hernia: open versus thoracoscopic surgery". Pediatric surgery international 29.11 (2013): 1183-1186.

15. Cho SD., et al. "Analysis of 29 consecutive thoracoscopic repairs of congenital diaphragmatic hernia in neonates compared to historical controls". Journal of pediatric surgery 44.1 (2009): 80-86.

16. Arca MJ., et al. "Early experience with minimally invasive repair of congenital diaphragmatic hernias: results and lessons learned". Journal of pediatric surgery 38.11 (2003): 1563-1568.

17. Gander JW., et al. "Early recurrence of congenital diaphragmatic hernia is higher after thoracoscopic than open repair: a single institutional study". Journal of pediatric surgery 46.7 (2011): 1303-1308.

18. Jancelewicz T., et al. "Thoracoscopic repair of neonatal congenital diaphragmatic hernia (CDH): outcomes after a systematic quality improvement process". Journal of pediatric surgery 48.2 (2013): 321-325.

19. Tyson AF., et al. "Thoracoscopic Versus Open Congenital Diaphragmatic Hernia Repair: Single Tertiary Center Review". Journal of Laparoendoscpic andAdvanced Surgical Techniques 27.11 (2017): 1209-1216.

20. Lacher M., et al. "Thoracoscopic $\mathrm{CDH}$ repair-A survey on opinion and experience among IPEG members". Journal of Laparoendoscpic and Advanced Surgical Techniques 25.11 (2015): 954-957.

Volume 2 Issue 9 September 2019

(C) All rights are reserved by Ali Raza Brohi and Naseem Mengal. 